\title{
Super-Resolution using Anchored Neighborhood Regression
}

\author{
T.Madhulatha, Gowri
}

\begin{abstract}
Starting late there have been enormous advances in picture up scaling or picture super-objectives reliant language of less and imperative benchmarks models. The organization time of to the systems is regularly disregarded paying little notice to how it is an essential thing for guaranteed application. This paper propose shrewd great-targets techniques while manufacture no exchange off on superiority. Regardless, we reinforce the utilization of hopeless insightful word references in mix in with national embedding's methods. For this condition, nearest neighbor are figured using the association from language particles slightly than the Euclidean section. Furthermore, we show that most of to the present strategy land at top execution from the correct parameters. Second, we show that utilizing all in all network coding has extensive speed focal centers, diminishing the super-goals map to precomputed projective framework. Third, we propose asserted neighborhood regression. That is to remain the zone displaying of a low goals fix to the closest molecule in dictionary and to pre compute the relating implanting mother trix. These recommendations are showed up distinctively in relationship with current top level strategies on standard pictures. We obtain in every way that really matters unclear or improved quality and a couple of offers of monstrosity speed updates

Keywords: neighborhood regression, focal centers
\end{abstract}

\section{INTRODUCTION}

Super-declaration is an outstanding part of picture re-development a spotlights on to the improvement of picture re-goals. Ordinarily, it takes at least one low-goals (LR) pictures as info and map as a high-goals (HR) yield picture. great-goals calculations may be generally partitioned at three subcategories: addition strategies, for example, Lanczos up sampling [5] and NewEdge Direct Interpolation (NEDI) [10], multi-outline techniques $[6,7,11]$ to create a high-resolution image of multiple frames of a single scene. Being nicknamed, and learning-oriented techniques. Subsequent uses include machine learning techniques and methods such as the slope profile earlier [13], which endeavor to learn normal to-edge insights

Revised Manuscript Received on December 22, 2019.

T.Madhulatha, Department of ECE, Malla Reddy Engineering College for Women, Telangana, India Engineering College for Women, Telangana, India
Gowri, Department of ECE, Department of ECE, Malla Reddy

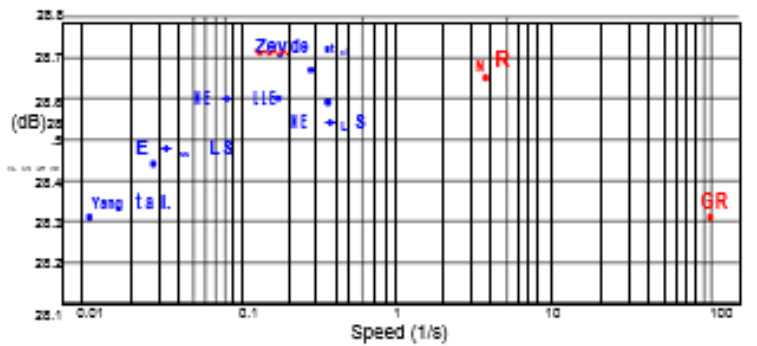

Figure 1. Fast versus PSNR for the attempted strategies.

Our ANR \&GR techniques (showed up in red) give together fast and quality. Extra nuances in Table 1.

Pictures, anyway later and understood dictionary or diagram based learning systems. An enormous segment of these dictionary set up together methods are based as for created by Freemanet al. [8] and Baker\&Kanade [2]. Word reference base systems utilize a fix or highlight based methodology to manage choose the association between nearby picture decals in low objectives and significant standards versions of a comparative view. The data picture is ordinarily secluded into covering area, which jointly structure the MarkovRan-Dome Field (MRF) system. By methods for checking for the closest neighbor in a low-objectives dictionary, you can find many candidates with high resolution. This is an MRF with several HRup-and-comer area for every hub. The MRF is solved by applying methods such as trust propositions or graph cuts, after associating the cost of data for each candidate and the recurring cost for neighboring candidates. One drawback of these strategies is their high computational multifaceted nature. A few techniques have been proposed to defeat this issue, most eminently the neighboring implant ding and little programming methodologies. Neighbors installed super-goals strategies [4, 3] don't generally concentrate on diminishing computational intricacy, yet they can be utilized to lessen the quantity of picture fix avoidance because of the innate introduction of the fix subspace. Handymen are required, in this manner lessening the execution time of the calculation. Short coding techniques [17, 18] endeavor to discover little coding for a input areae reliant on to a limited vocabulary (made by apply $\mathrm{K}$-ways or similar counts to planning Area). We give a review of these techniques in Sec. 2. Bevilacquaet al. [3] evaluate the operation time for a few, model base great-objectives counts, dependent upon the size of the data picture, from minutes to hours for generally top tier procedures. 


\section{Super-Resolution using Anchored Neighborhood Regression}

We propose another technique for example set up together super-objectives that fixations as for shorter estimation time while keeping up the abstract execution generally top tier upproaches. achieve an ordinary developedin speed some place in the scope of one and two arrangements of degree over other top tier method rest of to the paper is filtered through as looks for after: In Section 2 we study advancing procedures for national install decoding and little coding for superdestinations, we delineate our proposed system in Section 3, \& shows fundamental accomplishes Section 4. Completions are pulled in Section 5.

\section{II. LEXICON BASED SUPER-RESOLUTION}

At segment we rapidly diagram word reference base methods for SR. the promptly demonstrated Freeman's exceptional technique [8] in to the presentation, here base on national embeddings and lacking coding moves close.

\subsection{Neighbor installing draws near}

National implanting (NE) consider recognize that little picture area from low goals picture $\&$ its high rearrangement assistant structure low dimensional nonlinear manifold equivalentneighborhood geometry Change et al. [4] star addressed a superdestinations framework dependent on to this standard, using the mind boggling knowledge system nearby LinearEmbedding(LLE) [12]. LLE calculation expect that when enough models are accessible, each model \&neighbors lie on or close to a local straight fix of to the complex. Since manifold inLR \& HRjoin space are assume to has a comparable near to geometry, this proposes as long enough models are accessible, fixes in to the HRinclude locale could be aerated up as a biased run of the mill of near to neighbors utilizing dubious weights from in theLR feature space. Change. channel for a huge amount of $\mathrm{K}$ closest national for each datum fix inLR highlight space, compute $\mathrm{K}$ fitting for mimicking theLR fix by result an obliged least squares strategy, and event-associate make a HRfix by apply these stacks in HRjoin space. The outcome picture is a made by utilizing the figured HRfixes and average their obligations where they spread. The constant Non negative national Embedding move toward [3] is another occasion of $\mathrm{NE}$ utilized for grate-destinations. It depends upon the uncertainty that the local non negative least square rot stacks over the near to neighborhoods.

\subsection{Meager coding draws near}

The NE come nearer with the past area utilize a dictionary of tested area from low \&high goals picture sets. This word references can immediately turn out to be enormous, particularly when more or greater preparing pictures are add to develop execution. Meager coding consider attempt to conquer by utilizing an educated minimized word reference dependent on inadequate sign portrayal. Yang et al. [17] propose a methodology for supercoils dependent on to this thought. Low goals area are inadequately remade a scholarly word reference utilizing the accompanying detailing:

$$
\min \|F \mathbf{D} \alpha-F \mathbf{y}\|_{\mathbf{2}}+\lambda\|\boldsymbol{\alpha}\|_{\mathbf{0}},
$$

When $\mathrm{F}$ is a component removal administrator, $\mathrm{Dl}$ is adapted low goals word reference, $\alpha$ is scanty portrayal, $y$ is a short goals input fix and $\lambda$ is a weightings aspect. The 10 -standard imperative prompts a NP-difficult issue and, practically speaking, is loose to an 11-standard requirement. Condition
(1) is in the end additionally reached out with a term which empowers likeness in the covering districts of close by area. Inadequate word references are mutually learning for short and significant standards picture area, with the goal of have to the identical small depiction for low objectives fixes on the corresponding significant standards area. This goal is followed a set of planning picture fix sets $\mathrm{Xh}, \mathrm{Yl}$ by restricting

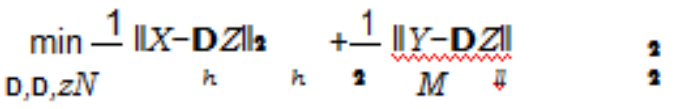

$$
\begin{aligned}
& +\lambda\left(\frac{1}{N}+\frac{1}{M}\right)\|Z\| \text {, }
\end{aligned}
$$

$\mathrm{H}$ ere $\mathrm{N} \& \mathrm{M}$ denotes dimensional low \& huge principles area $\& \mathrm{Z}$ represents a coefficient vector address a sparsely fundamental. A subsequent jargon had a particular size \& thusly figuring had become the farthest point of taking from arranging area while evading long virtuoso halting occasions because of a regularly making word reference. Disastrously, understanding this inadequate models still take a lot of time.

\section{Proposed Methods}

We had proposed an anchoredneighborhood reversion strategy which passes on 2 circumstances, one was a general conduct there theneighborhood structure was set \& another being an alleged Globalcase, where aneighborhood co-lists with an entire word reference being used. They allude to these in an after as an Anchoredneighborhood Regression \& its outrageous situation, a GlobalRegression. We have begun with a Globalcase to straightforwardness for a definition, and afterward we think about a neighborhoods.

\subsection{Global Regression}

For almost NE \& SC draws near, a least squares (LS) issues were compelled or normalized utilizing 11-standard of a coefficients, like condition (1). This is computationally requesting. We will reformulate a problem as the minimum squares regression normalized with a 12-standard of a coefficients. In this way, we utilize the Ridge Regression [14] have shut structure arrangement. An issue becomes

$$
\min \|\mathbf{y} F-\mathbf{N} l \boldsymbol{\beta}\|^{2}+\lambda\|\boldsymbol{\beta}\| 2,
$$

\section{$\beta$}

where $\mathrm{Nl}$ relates to a region inLR space that we decide to tackle this issue, which for a situation ofneighborhood implanting would allude to a $\mathrm{K}$ closest neighbors of an info highlight $\mathrm{yF}$ and for a situation of meager coding would allude to aLR lexicon. A parameter $\lambda$ permits us to ease a peculiarity (not well presented) issues and settles an answer, which is a coefficient vector $\beta$. An arithmetical arrangement is given by

$\boldsymbol{\beta} l=(\mathbf{N} T \mathbf{N} l+\lambda \mathbf{I})^{-} 1 \mathbf{N} T \mathbf{y} F l$.

HR patches will be computed using same coefficients on high resolution neighborhood $\mathbf{N} h$

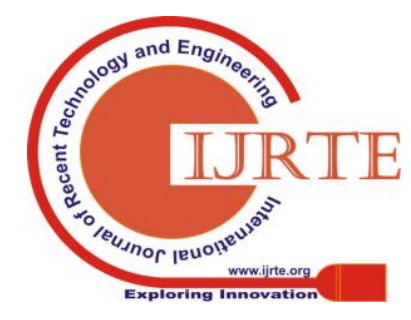


$\mathbf{x}=\mathbf{N} h \boldsymbol{\beta}$,

Where $\mathbf{x}$ is HRoutput patch \& $\mathbf{N} h$, HRneighborhood corresponding to $\mathbf{N} l$.

If we utilize wholeLR dictionary to this, meaning $(\mathbf{N} h, \mathbf{N} l)$ $=(\mathbf{D} h, \mathbf{D} l)$, we get Globalsolution to problem. An important observation here is that from equation (4) \& equation (5), we obtain:

$$
\mathbf{x}=\mathbf{D} h\left(\mathbf{D}^{T} \mathbf{D} l+\lambda \mathbf{I}\right)^{-} 1 \mathbf{D}^{T} \mathbf{y} F
$$

\subsection{Anchoredneighborhood Regression}

The GlobalRegression approach diminishes a super-goals procedure to the projection of each information include into a HRspace by augmentation with the precomputed grid. It is an anyway the Globalarrangement and therefore not tuned towards explicit information highlights, but instead whole lexicon, which is the portrayal of a highlights happening in a preparation pictures. On the off chance that as opposed to considering an entire lexicon as beginning stage for registering a projective framework we consider nearby neighborhoods of the given size we permit greater adaptability of approach to a detriment of expanded calculation - we will have more than one projective lattice and neighborhoods.

We begin by gathering a word reference molecules into neighbor-hoods. All the more explicitly, for every molecule in a word reference we process its $\mathrm{K}$ closest neighbors, which will speak to its neighborhood. On the off chance which we start from the scholarly inadequate word reference, as in a sparsity implements to Zeydeet al \& Yang et al. [17] [18], we discover a closest surrounds dependent on a relationship among the lexicon particles as opposed to an Euclidean separation. An explanation behind this is a that an iotas are the educated premise comprising of 12-standardized vectors. Assuming, on the other hand, we have the lexicon of highlights taken directly from a preparation patches, as in a NE approaches of Bevilacqua et al. \& Chang et al. [4] [3], at that point an Euclidean separation is an a fitting separation measure. When a neighborhoods are de-fined, we can figure the different projection grid $\mathrm{Pj}$ for every word reference iota $\mathrm{dj}$, in light of its own neighborhood. This should be possible in an equivalent manner as in a past area by utilizing just a lexicon particles that happen in aneighborhood instead of a whole word reference, and can again be registered disconnected.

The super-goals issue would then be able to be unraveled by figuring for each info fix include yiF its closest neighbor iota, $\mathrm{dj}$, in a word reference, trailed by a mapping to HRspace utilizing a put away projection framework $\mathrm{Pj}$ :

$$
\mathbf{x} i=\mathbf{P} j \mathbf{y} i F .
$$

This is the nearby estimate of a NE approach, with the low unpredictability \& thus the huge improvement in execution time. We consider our methodology an Anchored Neighborhood Regression, since a neighborhoods are anchored to a lexicon iotas \&not legitimately to a low goals fixes as in another NE draws near.

\section{EXPERIMENTS}

In the present segment we reveal exploratory results 1 of strategy \&compare numerically \&qualitatively to another condition of workmanship techniques. They initially examine a portion of a subtleties encompassing a calculation, for example, utilized highlights, word reference decisions, similitude measures, size forneighborhood count \&different fix embedding's..
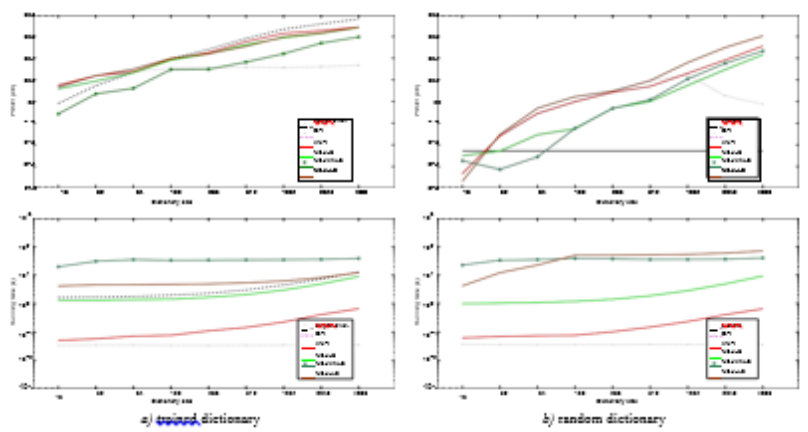

Figure 2. Dictionarysize vs.average PSNR \& average running timeperformance on 14 figures from Set14 with maximation $3 \times$. Bicubicis our reference.

Every one of all techniques were utilized with their bestneighborhood shape. The prepared word references, ANR utilizes the structure of 40, The NE+LS utilizes 12, NE+LLE-24 NE+NNLS-24. For an irregular word references, ANR utilizes the size of 128, NE+NNLS-24, NE+LS-5, NE+LLE- 28, resp. We have used to denote a run occasions we subtracted a mutual handling time for each of all techniques.

\subsection{Conditions}

\subsubsection{Features}

One angle which can impact $\mathrm{Z}$ execution is a $\mathrm{Z}$ sort of highlights used to this speak to $Z$ picture patches. These highlights are quite often determined from $\mathrm{Z}$ luminance part of $\mathrm{Z}$ picture, while $\mathrm{Z}$ shading segments are inserted utilizing the standard addition calculation, for example, Bicubicintroduction $[9,18,17,4,3]$. That used on the grounds that $\mathrm{Z}$ human effort framework is a considerably decrease delicate to this large recurrence shading changes than high recurrence power variations, so they are used for $\mathrm{Z}$ amplification factors utilized in the major papers $\mathrm{Z}$ saw contrast between Bicubicintroduction and SR of $\mathrm{Z}$ shading channels is an insignificant.

The most essential component to this utilization is a $\mathrm{Z}$ fix itself. This anyway doesn't give $\mathrm{Z}$ include great speculation properties, so the well known decision is a subtract $Z$ mean [3] and to this standardize $\mathrm{Z}$ differentiate [8] by for example isolating by $\mathrm{Z}$ standard deviation. A regularly utilized comparative element is a $\mathrm{Z}$ first-and second request subordinate of $\mathrm{Z}$ fix $[4,17,18]$.

Both of the above element kinds appear to this approach to the comparative execution, when Bevilacqua et al. [3] reveal which utilizes just first or-der subordinates provides somewhat more regrettable execution than utilizing just mean subtraction. We utilise $\mathrm{Z}$ same highlights as Zeydeet al. [18], who begin as $Z$ first-\& second request slopes \& implement PCA dimensionality decrease, anticipating $Z$ highlights onto the low-dimensional subspace when pre-serving $99.9 \%$ of $Z$ normal vitality. That normally prompts this highlights of around 30 measurements for upscaling factor $3 \& 3 \times 3$ low goals fix sizes. 


\section{Super-Resolution using Anchored Neighborhood Regression}

We subtract $\mathrm{Z}$ bicubically interjectedLR picture from $\mathrm{Z}$ HRpicture to this make standardized HRpatches. $Z$ patches coming about because of Z SR process (for example condition (6) for GR) are added to this $\mathrm{Z}$ bicubically addedLR input picture (with covering parts arrived at the midpoint of) to this make $\mathrm{Z}$ yield.

We use Zeydeet al's. Calculation and their sources as the beginning stage for our executions.

\subsubsection{Embeddings}

Aside from our examinations with an inadequate techniques for Yang et al. [17] and Zeydeet al. [18], we likewise contrast our outcomes with be neighbor inserting approaches adjusted to be our word reference decisions. a unique LLE-based SR strategy for Chang et al. [4] doesn't utilize the scholarly word reference, in-stead a lexicon comprises just of a preparation patches themselves. This makes direct correlation with be our strategy and a meager techniques troublesome, on the grounds that an inquiry at that point emerges "which lexicon would it be a good idea for us to use to be have the reasonable com-parison?". an equivalent can be said when we wish to be contrast with be a nonnegative neighbor implanting of Bevilacqua et al. [3]. an answer is a to be utilize an equivalent educated word reference as Zeydeet al. what's more, our strategy, with a separate SR meth-ods of Chang and Bevilacqua actualized to be explain a SR regression. We will allude to be these as NE + LLE (Neigh-bor Embedding with Locally Linear Embedding) and NE.
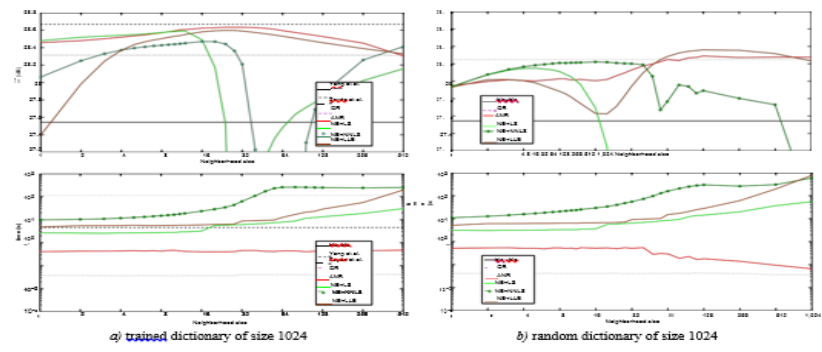

Figure 3.neighborhood structure versus normal PSNR \& normal running time implementation on a 14 pictures obtained with Set14 with amplification $\times 3$. Zeydeet al Bicubic, \& GR Yang et al., ., are accounted to get base value. The scientist Yang et al utilizes a unique word reference of 1022, while a different strategies, aside from Bicubicinsertion, share an equivalent lexicon.

\subsubsection{Dictionaries}

The decision of a word reference is a basic for a presentation of any SR technique. Typically, a bigger a word reference a superior a presentation, anyway this accompanies the higher computational expense. A word reference can be fabricated utilizing aLR input picture themselves, in situation we had an "inner" lexicon. Glasner et al. [9] \& his serious exploita-tion of "fix excess" is a primary promoters for this. Nonetheless, numerous methodologies like to be assemble "outside" dic-tionaries, outer to be an info question, utilizing differing pictures. In the settings we work with an equivalent arrangement of outer pictures as utilized by Zeydeet al. [18] and Yang et al. [17]. Likewise, we consider both arbitrarily inspected word references and learned lexicons. For learning we utilize a K-SVD/OMP learning approach of Zeydeet al. [18]. In Fig. 2 we delineate an impact of a word reference on a for each formance. True to form, generally an exhibition increments with a size of a lexicon. In addition, we see again that utilizing the educated word reference is an exceptionally valuable for every one of a strategies it takes into account the sensibly superior for little lexicon sizes. One needs the $16 \times$ bigger arbitrary inspected word reference to be arrive at an equivalent presentation similarly as with a prepared lexicon. The vast majority of a strategies display the sim-ilar log-direct expanding pattern with a word reference size and a PSNR contrast among ANR, Zeydeet al. what's more, a NE approaches is a very little for their best settings (utilizing operation timalneighborhood size). A distinction is a made by a running time, where ANR and GR are a reasonable champs. GR is the quickest strategy, yet as the Globaltechnique it has its shortcomings, and for huge word references tends not to be arrive at focused PSNR levels.

\subsubsection{Neighborhoods}

As clarified in the Section 3.2, our ANR calculation finds a closest neighbor in a word reference for each information feature and gets aneighborhood and a precomputed projection grid from this neighbor. NE approaches likewise depend on aneighborhood to be an informationLR highlight. A presentation of an installing techniques, \& thus for each formance of a SR strategy, relies upon a dimensionality from those surrounds.

The calculation of a closest neighbors is a dependent on the likeness measure. Euclidean separation is the decision of most NE approaches working legitimately with enormous dictionaries. We utilize Euclidean separation for an arrangements with haphazardly inspected word references. in the an instance of a scholarly scanty lexicons, we acquire 12-standardized molecules intended to be structure the premise spreading over a space of a preparation tests while limiting a recreation mistake. For this case our alternative is a acquire a closest neighbors utilizing a correlation communicated as an inward item.

Table 1. Amplification $\times 3$ execution in the terms of PSNR $(\mathrm{dB})$ and running time (s) per picture on a Set14 dataset. Each of the a unique techniques utilize an equivalent preparing pictures from [17]. a strategies share an equivalent prepared word reference of 1024, with the exception of Bicubicintroduction and Yang et al. with the lexicon of 1022. aneighborhood sizes are as in the Fig. 2. ANR is a 5 times quicker than Zeydeet al. On the off chance that we consider just an encoding time, our ANR technique takes $0.27 \mathrm{~s}$ all things considered, being multiple times quicker than Zeydeet al., and multiple times quicker than NE+LS.

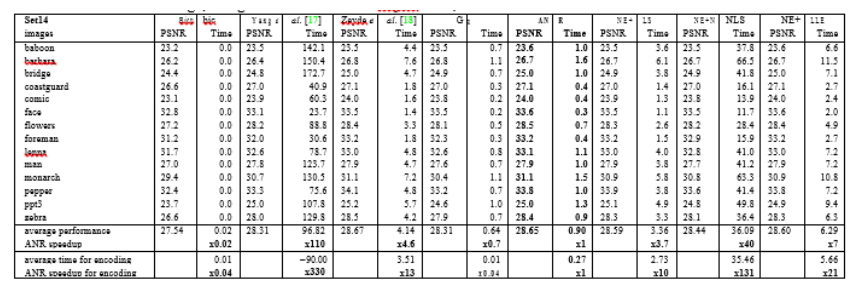

Aneighborhood size is significant parameter for a NE procedures and for ANR also. We show an impact of this size in the Figure 3 for word references of size 1024. 
A strategies carry on diversely under an equivalent settings. In addition, a bends are not monotonic - as saw likewise in [3] and more examination in the marvel is a due. On a scholarly dictionary, NN + LS tops at 12, NE + LLE and NE + NNLS at 24, while ANR tops at 40. On an arbitrary word reference,

$\mathrm{NN}+\mathrm{LS}$ tops at 5, NE + LLE at 128, NE + NNLS at 24, while ANR tops at 128 . We will utilize theseneighborhood sizes for a further analyses. A conduct of ANR and GR is a likewise impacted by a decision of a regularization parameter $\lambda$, in the every one of our investigations exactly set to be 0.01 .

\subsection{Performance}

In the present segment we would exhibit quantitative \& subjective outcomes just which as running occasions applied to the proposed technique \& contrast with each other examined word reference referenced SR calculations. All explicitly, we contrast for our outcomes with be an inadequate coding calculations according to Zeydee \& Yang et al. [17] [18], just as to be our usage dependent on a LS regressions utilized by Chang et al. [4] (NE + LLE) \& Bevilacqua et al. [3] (NE + NNLS) that as depicted in the Sec-tion 4.1.2. Tables $1 \& 2$ abridge an outcomes, demonstrating PSNR and running time esteems for the quantity of test pictures. a pictures are isolated into 2 sets; Set14 utilized by Zeydeet al. to be reveal their outcomes and Set5 that utilized with Bevilacqua et al. an impact of word reference size is an investigated in the Fig. 2, when Fig. 3 reveals a connection among neigh-borhood structure, PSNR \& time.

\subsubsection{Quality}

While utilizing an idealneighborhood size for every technique a PSNR of Zeydeet al. [18], NE + LLE, NE + LS, \& our ANR technique arrive at practically identical normal qualities. A methodology of Zeydeet al. arrives at most noteworthy PSNR in the all tests, somewhat over our ANR strategy. On the Set14 dataset Zeydeet al. achieves a normal of $28.67 \mathrm{~dB}$, while ANR gets $28.65 \mathrm{~dB}$, and NE + LS \& NE + LLE provide a common of $28.6 \mathrm{~dB}$. Our GR strategy and Yang et al. get an equivalent normal PSNR of $28.31 \mathrm{~dB}$, while NE + NNLS lies among $28.44 \mathrm{~dB}$. The comparable conduct will be observed as a Set5 database, there ANR could be superior to Zeydeet al. Visual models were appeared in the Figures $4,5, \& 6$. From those we could reason which ANR provides fundamentally the same as quality presentation as a top strategies it was contrasted with.

\subsubsection{Running Time}

The usage of NNLS was comparative calculation time as that is an announced by Bevilacqua et al. [3], that is an in the a lot of 10 seconds for the amplification factor of $3 \times$. That could likewise be seen in the Figure $2 \&$ Figure 3 . We would com-pare with their calculation since it was a exceptionally ongoing strategy focused on low unpredictability and high preparing velocity while as yet keeping top notch results, and is a thusly a perfect possibility for reference.

At the point when we look at a handling times it is a reasonable that our GlobalRegression calculation is an a quickest by a wide margin, fol-lowed by our Anchoredneighborhood Regression. a last line of Table 1 just as Fig. 2 and 3 show a distinction of an encoding time, which is an a preparing time that is a left in the wake of subtracting a mutual handling time of 0.63 sec-onds of a calculations (pre/post preparing, Bicubicbetween polation, fix extraction, and so on.).
Table 2. Magnification $\times 2, \times 3$, and $\times 4$ execution in the terms of PSNR (dB) and running time (s) per picture on a Set5 dataset. Every one of the a unique techniques utilize an equivalent preparing pictures from [17]. Each of the a strategies share an equivalent prepared word reference of 1024, with the exception of Bicubicintroduction and Yang et al. with the lexicon of 1022. We utilize an equivalentneighborhood measures as in the Fig. 2. For upscaling factor 3, ANR is a 5 times quicker than Zeydeet al. being multiple times quicker than Yang et al. what's more, multiple times quicker than NE+LS with 12 neighbors

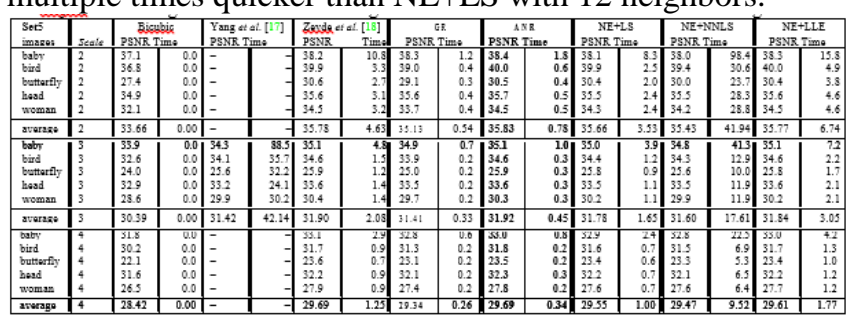

ANR approach for speed enhancements of $13 \times, 330 \times, 21 \times$, $\& 131 \times$, resp.

\section{RESULTS}

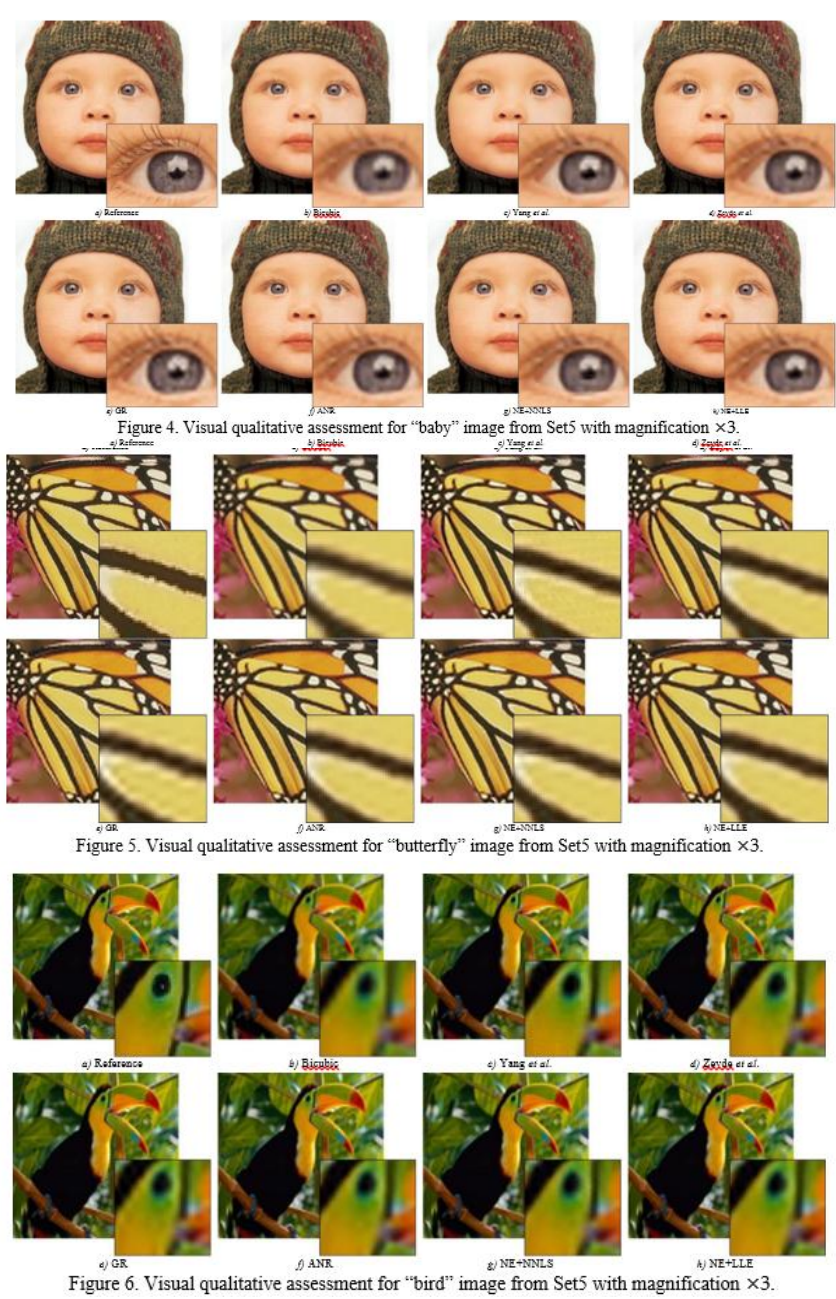

Figure 6. Visual qualitative assessment for "bird" image from Set5 with magnification $\times 3$. 


\section{Super-Resolution using Anchored Neighborhood Regression}

\section{Conclusion}

We have proposed a new technique referenced strategy used for achieving super-goals known as Anchored Neighbor Reversion that centers around quick execution while holding a subjective execution of late best in class techniques. We additionally professional represented an outrageous variation of this known as GlobalRegression that centers absolutely around high execution speed in the trade for some visual quality misfortune. a principle commitments of that paper which are twofold: I) we have implemented an ANR method, which uses edge regression to be study the model surrounds disconnected and utilizes those surrounds to be precompute projections will be mapLR patches into a HRspace, \& ii) we appear through our investigation of existingneighborhood insert ding SR techniques that a large portion of these can arrive at the comparative top execution dependent on utilizing a fittingneighborhood size and lexicon; an inadequate scholarly word references in the mix with neighbor installing's strategies were demonstrated to be the quicker choice to be full scanty coding strategies.

We intend to be stretch out our technique to be utilize an additional element of time for an instance of video successions, with ongoing gushing super-settled video as the objective.

\section{REFERENCES}

1. M. Aharon, M. Elad, and A. Bruckstein. K-SVD: an algo-rithm for designing overcomplete dictionaries for sparse rep-resentation. IEEE Trans. on Sig. Proc., 54(11), 2006. 2

2. S. Baker and T. Kanade. Hallucinating faces. In Automatic Face and Gesture Recognition, 2000. 1

3. M. Bevilacqua, A. Roumy, C. Guillemot, and M.-L. Al-beri Morel. Low-complexity single-image super-resolution based on nonnegative neighbor embedding. In BMVC, 2012. 1,2,3,4,6

4. H. Chang, D.-Y. Yeung, and Y. Xiong. Super-resolution through neighbor embedding. CVPR, 01:275-282, 2004. 1, 2,3,4,6

5. C. E. Duchon. Lanczos Filtering in One and Two Dimen-sions. J. Appl. Meteorology, 18:1016-1022, 1979. 1

6. S. Farsiu, M. D. Robinson, M. Elad, and P. Milanfar. Fast and robust multiframe super resolution. Trans. Img. Proc., 13(10):1327-1344, Oct. 2004. 1

7. R. Fransens, C. Strecha, and L. Van Gool. Optical flow based super-resolution: A probabilistic approach. Computer Vision and Image Understanding, 106(1):106-115, 2007. 1

8. W. T. Freeman, E. C. Pasztor, and O. T. Carmichael. Learn-ing low-level vision. International Journal of Computer Vi-sion, 40(1):25-47, 2000. 1, 2, 4

9. D. Glasner, S. Bagon, and M. Irani. Super-resolution from a single image. In ICCV, 2009. 4, 5

10. X. Li and M. T. Orchard. New edge-directed interpolation. Trans. Img. Proc., 10(10):1521-1527, 2001. 1

11. M. Protter, M. Elad, H. Takeda, and P. Milanfar. Generaliz-ing the non-local-means to super-resolution reconstruction. In Trans. Img. Proc., 2009. 1

12. S. Roweis and L. Saul. Nonlinear dimensionality reduction by locally linear embedding. In ICCV, 2001. 2

13. J. Sun, Z. Xu, and H.-Y. Shum. Image super-resolution using gradient profile prior. In CVPR, 2008. 1

14. A. N. Tikhonov and V. Y. Arsenin. Solution of Ill-posed Problems. Winston \& Sons, 1977. 3

15. R. Timofte and L. Van Gool. Adaptive and weighted col-laborative representations for image classification. Pattern Recognition Letters, 2013. 3

16. J. A. Tropp and A. C. Gilbert. Signal recovery from ran-dom measurements via orthogonal matching pursuit. IEEE Transactions on Information Theory, 53(12), 2007. 2

17. J. Yang, J. Wright, T. Huang, and Y. Ma. Image super-resolution via sparse representation. Image Processing, IEEE Transactions on, 19(11):2861-2873, 2010. 1, 2, 3, 4, 5, 6, 7

\section{AUTHORS PROFILE}

T.Madhulatha, Department of ECE, Malla Reddy Engineering College for Women, Telangana, India

Gowri, Department of ECE, Department of ECE, Malla Reddy Engineering College for Women, Telangana, India 